\title{
Gaming Controller Emulator
}

\section{(Customizable android application as an alternative to gaming controller hardware using wireless communication and socket programming)}

\author{
Yogesh Nawale ${ }^{1}$, ShaileshNighojkar ${ }^{2}$, Yogesh Patil $^{3}$, SagarSatpute ${ }^{4}$ \\ Student, BE Comp, PVG's COET, Pune, India ${ }^{1,2,3,4}$
}

\begin{abstract}
In the video gaming world, controller enhances game play but requires to purchase controller hardware exclusively, as an accessories input device. Although the gaming controller hardware has its own importance, this project gives mobile device running android operating system as an alternative to it. To simulate the idea, Client-Server architecture is used where server is on computer running the video game and client is android mobile application. User can select an xml profile which contains information regarding controller screen interface and corresponding input values from input sensors. These profiles are predefined or can be user defined by customizable wizard. Values from sensors are quantized and then passed to server using Wi-Fi connectivity and socket programming then Server injects values to video game. Thus, this android app performing as an input device gaming controller to computer.
\end{abstract}

Keywords: Android, Client-Server Architecture, XML, Wi-Fi, Socket, Configuration File.

\section{INTRODUCTION}

PC controllers are the devices or software which controls PC using client-server architecture. Where devices like gaming pad or software like virtual pc remote controller are act as client and computer running game, presentation etc. act as server. Game pad enhances gameplay with extra dedicated peripheral to control game action. Although having such advantage, dedicated hardware gives extra baggage of purchasing it whereas virtual pc controller gives freedom of remote access and no need of extra dedicated hardware.

Various video games requires controllers for better experience such controllers can be created virtually on mobile device as android mobile application and connected to pc through wireless connectivity. Generally fixed controller Setting restricts from full gaming experience comfort level because setting is hard coated by plastics buttons on it. Such restrictions can be avoided by software approach with gives freedom of customizability. In addition game action can be controlled by mobile sensors such as magnetic sensor, rotation sensor, gyroscope etc. and input is given to computer by quantizing the outputs from senores reading and giving it as an event occurred like key press events, mouse events which is in short simulation of input events to the running gaming windows computer machine, various gaming controller categories can be implemented through it like game pad, steering wheel, gun, joystick etc.

To achieve this concept android mobile application is created. A server is created on machine running game i.e. windows and client(s) are created on android mobile device(s) and $\mathrm{Wi}-\mathrm{Fi}$ wireless communication is used to transfer of information in between. Configuration files are customized to configure the requirements of controller functionality which can be newly created and shared over the community. Such a three-tier architecture is used in which presentation tier is controlled running game with custom controller UI and logic tier is event management of controller, communication strategies used and data tier is $\mathrm{xml}$ configuration file which is on android device.

\section{SYSTEM BLOCK DIAGRAM}

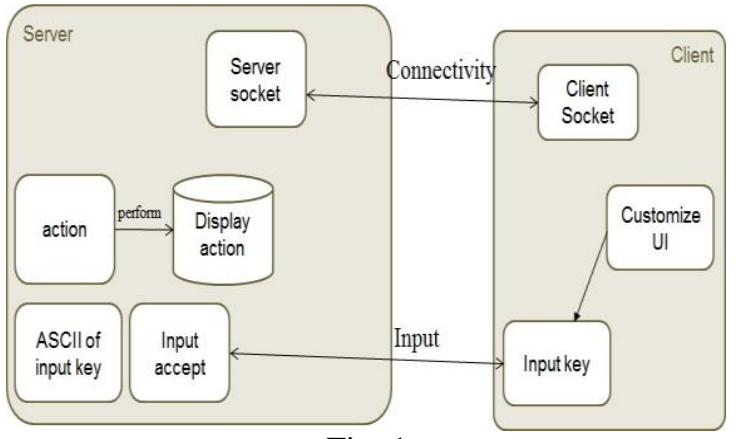

Fig. 1

\section{SYSTEM ARCHITECTURE AND WORKING}

1. Connecting and loading Controller:

Controller-Receiver/Injector communication is established by TCP connection, connectivity information is manually entered or auto connected by cached data from history connection. After successful connection establishment, Controller selects configuration file from available list and then validates it for compatibility. Device supporting features listed can only be able to load configuration file.

2. Synchronization with server:

Event Handling data is synchronized with server in order to reduce the traffic bandwidth, multiple events generated can be clubbed together and then transferred as single integer toserver and using synchronization mapping available with server it can then use for decode individual event and then injection.

3. Separateevent handling for ensuring injection to titled window:

To ensure game is at the front in process dictionary or it is a focused window, a separate thread provided as interrupt service, which interrupts main module when game loses or gains focus to avoid unnecessary injection.

4. Injection:

It generates a virtual input in system as if the event has happed. 
5. Configuration Creation steps:

a. Open new Configuration file wizard.

b.Start game on computer (receiver/server).

c. Basic info about game is auto filled and then manually contents or widgets are added onto screen forms acontroller.

d. Respective event handling information is simultaneously registered. Configuration file contains mapping of device event handling to game event handling such as accelerometer tilt to mouse movement in game.

\section{FEATURES}

List of some predefined features to add in configuration file:

1) Button/Area Press-release:

Particular area on controller screen is dedicated to handle touch events.

2) Sensor action:

Sensor actions such as tilt, rotation, acceleration used to manipulate events.

3)Cheat helper:

Cheats can directly inserted with one button press.

4)Driving module:

Dedicated driving module for racing games which contains steering wheel, break, acceleration, gear pad etc. for racing game.

5)Gun module:

Accelerometer moves mouse and move button used for movement and fire. Accelerometer/gyroscope use to calculate tilt and mouse action.

\section{CONCLUSION}

Currently in the Gaming world there exists some third party hardware gaming consoles to provide support for gaming. But the main disadvantage of such third party hardware consoles is that they are hardware's which might be hard to carry everywhere but portable mobile phones having android OS are easy to carry everywhere, and also the mobiles are easy to handle. And above that, a sensors which are added after which buttons will not be required to control cars and moving actions. This process is quite tedious for a normal user first. Hence there is a necessity to add Customizable User Interface which gives better help to in gaming.

Basically, the main aim behind implementing this project is to replace the third party hardware consoles into software console. So on the basic manner, it is expected that game action can be controlled by mobile sensors such as magnetic sensor, rotation sensor, gyroscope etc. and input is given to computer by quantizing the outputs from senores reading and giving it as an event occurred like key press events, mouse events which is in short simulation of input events to the running gaming windows computer machine, various gaming controller categories can be implemented through it like game pad, steering wheel, gun, joystick etc.

Thus we expect from our application that it provide more comfort level while playing games than third party hardware console.

\section{REFERENCES}

[1]. Remote computer access through Android mobiles- Jaya Bharathi chintalapati1, Srinivasa Rao T.Y.S2 Department of Computer Science \& Engineering.

[2]. Virtual Network Computing, Tristan Richardson, Quentin StaffordFraser, Kenneth R. Wood and Andy Hopper, Reprint from IEEE Internet Computing Volume 2, Number 1 January/February 1998.FATvs. NTFS in a Forensic Environment - GIAC.

[3]. Remote Control of Mobile Devices in Android Platform Angel, Gonzalez Villan, Student Member, IEEE and JosepJorbaEsteve, Member, IEEE. 